\title{
Equivalences of Some Forms of the Change of Variable Formula and the Fundamental Theorem of Calculus
}

\author{
HARYONO TANDRA
}

Abstract. We discuss an interplay between some versions of the Change of Variable Theorem and the Fundamental Theorem of Calculus for the Riemann integral. We show that the two theorems are equivalent, and that for both theorems to be true it suffices to assume two particular formulas derived from them. In the realm of teaching, this material might be among our interests.

Key words and phrases: change of variable theorem, fundamental theorem of calculus.

ZDM Subject Classification: I20.

\section{Introduction}

This note discusses an interconnection between two (weak) versions of the Change of Variable Theorem for integrals (CVT) and the Fundamental Theorem of Calculus (FTC) for the Riemann integral. Here we assert that the FTC ([2], Theorem 2) and the two versions of CVT (the general version of the first is due to Kestelman [4] and that of the second one is due to Preiss and Uher [5]), imply one another. Also, we show that, for the FTC and all CVTs to be true it is enough to assume two particular formulas derived from them which, in a loose fashion, are:

(i) If $F:[a, b] \rightarrow \mathbb{R}$ is differentiable, nonzero, and $F^{\prime}$ is integrable with $\left|F^{\prime}\right| \geq \delta$ for some $\delta>0$ and $F^{\prime}$ has the same sign on $[a, b]$, then $F^{\prime} / F$ is integrable on 


$$
\text { "tmcs-tandra" — 2014/10/12 — 16:03 — page } 270 \text { — \#2 }
$$

$[a, b]$, and

(ii) If $0 \notin[a, b]$, then

$$
\int_{a}^{b} \frac{F^{\prime}(x)}{F(x)} d x=\int_{F(a)}^{F(b)} \frac{1}{y} d y
$$

$$
\int_{a}^{b} \frac{1}{x} d x=\ln |b|-\ln |a| .
$$

Our argument then provides an alternate approach of how a presentation of the teaching material about the theorems can be carried out with the following instructional gains: (1) it can interestingly be developed only from those particular formulas; (2) while only some elementary basic properties (we list them all) are needed, the theorems we concern can be presented in general forms in which the concepts of a set of measure zero and a Lipschitz condition for a function are used; and last but not least, (3) the proofs we present are independent from the Lebesgue criterion for the Riemann integrability.

Below we recall some basic concepts and related properties that we shall use.

(i) A real-valued function $f$ on $X \subseteq \mathbb{R}$ is said to be Lipschitz if there exists a positive number $M$ such that for all $s, t \in X$,

$$
|f(s)-f(t)| \leq M|s-t| .
$$

The number $M$ is called a Lipschitz constant for $f$.

(ii) A set $A$ of real numbers is said to have a measure zero if for any $\epsilon>0$ there exists a countable collection $\left\{\left(u_{n}, v_{n}\right)\right\}_{n=1}^{\infty}$ of open intervals such that $A \subseteq \cup_{n=1}^{\infty}\left(u_{n}, v_{n}\right)$ and $\sum_{n=1}^{\infty}\left(v_{n}-u_{n}\right)<\epsilon$.

(iii) Two functions $f$ and $g$ on $[a, b]$ are said to be equal almost everywhere, which we write $f=g$ a.e., if the set $\{x \in[a, b]: f(x) \neq g(x)\}$ is of measure 0 .

(iv) Let $[a, b]$ be a given interval, where $a<b$. By a partition $P$ of $[a, b]$ we mean a finite set of points $x_{0}, x_{1}, \ldots, x_{n}$, where $x_{0}:=a<x_{1}<\ldots<x_{n}:=b$. Suppose now $f$ is a bounded real function defined on $[a, b]$, and $P$ is a partition $x_{0}:=a<x_{1}<\ldots<x_{n}:=b$ of $[a, b]$ for which

$$
U(P, f):=\sum_{i=1}^{n} M_{i}\left(x_{i}-x_{i-1}\right) \quad \text { and } \quad L(P, f):=\sum_{i=1}^{n} m_{i}\left(x_{i}-x_{i-1}\right)
$$

where for each $i \in\{1, \ldots, n\}$,

$M_{i}:=\sup \left\{f(x): x_{i-1} \leq x \leq x_{i}\right\} \quad$ and $\quad m_{i}:=\inf \left\{f(x): x_{i-1} \leq x \leq x_{i}\right\}$. 


$$
\text { "tmcs-tandra" — 2014/10/12 - 16:03 — page } 271 \text { — \#3 }
$$

Now, let

$$
\begin{aligned}
& U(f):=\inf \{U(P, f): P \text { is a partition of }[a, b]\}, \\
& L(f):=\sup \{U(P, f): P \text { is a partition of }[a, b]\} .
\end{aligned}
$$

If $U(f)=L(f)$, we say that $f$ is Riemann integrable on $[a, b]$. In such a case we write $f \in \mathcal{R}[a, b]$ (that is $\mathcal{R}[a, b]$ denotes the set of all Riemann integrable functions on $[a, b])$ and we denote the common value by $\int_{a}^{b} f(x) d x$. In addition, we define $\int_{b}^{a} f(x) d x:=-\int_{a}^{b} f(x) d x$, and $\int_{a}^{b} f(x) d x:=0$ if $a=b$.

Throughout our discussion, we assume that all integrability are in the Riemann sense. The theorem below lists some basic properties we shall use.

\section{THEOREM 1.}

(i) The following properties hold for continuous and Lipschitz functions.

(a) If $f:[a, b] \rightarrow \mathbb{R}$ and $g:[c, d] \rightarrow \mathbb{R}$ are composable functions, then (1) if $f$ and $g$ are continuous, so is $f \circ g$; and (2) if $f$ and $g$ are Lipschitz, so is $f \circ g$.

(b) An indefinite integral $g_{c}(x):=\int_{c}^{x} f(t) d t$, where $c, x \in[a, b]$, is Lipschitz on $[a, b]$.

(c) If $f$ is Lipschitz on $[a, b]$, then $f$ is continuous on $[a, b]$.

(d) If $f$ is Lipschitz on $[a, b]$, then $f$ is uniformly continuous on $[a, b]$.

(e) If $f$ is continuous on $[a, b]$, then $f \in \mathcal{R}[a, b]$.

(f) [A weaker version of Theorem 14.13 in [1]] If $f$ is Lipschitz on $[a, b]$, and $A \subseteq[a, b]$ is of measure zero, then $f(A):=\{f(x): x \in A\}$ is of measure zero.

(ii) $f \in \mathcal{R}[a, b]$ if and only if for every $\epsilon>0$ there exists $\delta>0$ such that if $x_{0}:=a<x_{1}<\ldots<x_{n}:=b$ is any partition of $[a, b]$ with $x_{i}-x_{i-1}<\delta$, for all $i=1, \ldots n$, then for any $c_{i} \in\left[x_{i-1}, x_{i}\right]$,

$$
\left|\sum_{i=1}^{n} f\left(c_{i}\right)\left(x_{i}-x_{i-1}\right)-\int_{a}^{b} f(x) d x\right|<\epsilon .
$$

(iii) The following properties hold provided $f, g \in \mathcal{R}[a, b]$.

(a) For any $[s, t] \subseteq[a, b], f \in \mathcal{R}[s, t]$.

(b) [The additivity property over subintervals] If $z \in[a, b]$, then $\int_{a}^{b} f(x) d x=$ $\int_{a}^{z} f(x) d x+\int_{z}^{b} f(x) d x$. 


$$
\text { "tmcs-tandra" — 2014/10/12 - 16:03 — page } 272 \text { — \#4 }
$$

(c) [The linearity property] For any real number $\alpha$, we have $\alpha f, f+g \in$ $\mathcal{R}[a, b]$, where

$$
\int_{a}^{b} \alpha f(x) d x=\alpha \int_{a}^{b} f(x) d x
$$

and

$$
\int_{a}^{b}(f(x)+g(x)) d x=\int_{a}^{b} f(x) d x+\int_{a}^{b} g(x) d x .
$$

(d) $f \cdot g \in \mathcal{R}[a, b]$.

(e) If $f=g$ a.e., then $\int_{a}^{b} f(x) d x=\int_{a}^{b} g(x) d x$.

(f) [The monotonicity property] If $f(x) \geq g(x)$, for all $x \in[a, b]$, then $\int_{a}^{b} f(x) d x \geq \int_{a}^{b} g(x) d x$. In particular, if $f$ is integrable on $[a, b]$ and $f(x) \geq 0$, for all $x \in[a, b]$, then $\int_{a}^{b} f(x) d x \geq 0$.

(g) [Saks-Henstock Lemma for the Riemann integral] For every $\epsilon>0$ there exists $\delta>0$ such that if $x_{0}:=a<x_{1}<\ldots<x_{n}:=b$ is any partition of $[a, b]$ with $x_{i}-x_{i-1}<\delta$, for all $i=1, \ldots n$, then for any $c_{i} \in\left[x_{i-1}, x_{i}\right]$,

$$
\sum_{i=1}^{n}\left|f\left(c_{i}\right)\left(x_{i}-x_{i-1}\right)-\int_{x i-1}^{x_{i}} f(x) d x\right|<\epsilon .
$$

(For a general version see e. g. Corollary 5.4 in [1].)

We assume the following theorem to justify the fact that every Lipschitz function $f:[a, b] \rightarrow \mathbb{R}$ is differentiable except on a set of measure zero.

Theorem 2 (Rademacher). If $U$ is an open set of real numbers and $f: U \rightarrow$ $\mathbb{R}$ is Lipschitz, then $f$ is differentiable except on a set of measure zero.

It should be noted that we do not assume the following theorem.

Theorem 3 (Lebesgue criterion for the Riemann integrability). A function $f:[a, b] \rightarrow \mathbb{R}$ is Riemann integrable if and only if $f$ is bounded and $f$ is continuous except on a set of measure zero.

\section{The main theorem and remarks}

Let $F$ be a real-valued function on $[a, b]$, and $g$ be a real-valued function defined on $J:=F([a, b])$. Consider the following P1, P2, FTC, CVT 1, and CVT 2 : 
$\mathrm{P} 1$.

(i) If $F$ is Lipschitz, nonzero, and $F^{\prime}=f$ a.e., for some integrable function $f$ on $[a, b]$ where $|f| \geq \delta$ for some $\delta>0$ and $f$ has the same sign on $[a, b]$, then $f / F$ is integrable on $[a, b]$, and

$$
\int_{a}^{b} \frac{f(x)}{F(x)} d x=\int_{F(a)}^{F(b)} \frac{1}{y} d y
$$

(ii) If $0 \notin[a, b]$, then

$$
\int_{a}^{b} \frac{1}{x} d x=\ln |b|-\ln |a|
$$

P2. If $F$ is Lipschitz, nonzero, and $F^{\prime}=f$ a.e., for some integrable function $f$ on $[a, b]$, then $f / F$ is integrable on $[a, b]$, and

$$
\int_{a}^{b} \frac{f(x)}{F(x)} d x=\ln |F(b)|-\ln |F(a)| .
$$

FTC (Botsko, [2]). If $F$ is Lipschitz and $F^{\prime}=f$ a.e., for some integrable function $f$ on $[a, b]$, then

$$
\int_{a}^{b} f(x) d x=F(b)-F(a) .
$$

CVT 1 (A weaker version of Kestelman, [4]). If $F$ is Lipschitz, $F^{\prime}=f$ a.e., for some integrable function $f$ on $[a, b]$ where $f \geq 0$ (or $f \leq 0$ ), and $g$ is integrable on $J$, then $(g \circ F) f$ is integrable, and

$$
\int_{a}^{b} g(F(x)) f(x) d x=\int_{F(a)}^{F(b)} g(y) d y .
$$

CVT 2 (A weaker version of Preiss and Uher, [5]). Let $F$ be Lipschitz, and $F^{\prime}=f$ a.e., for some integrable function $f$ on $[a, b]$ where $|f| \geq \delta$ for some $\delta>0$ and $f$ has the same sign on $[a, b]$. If $g$ is bounded and $(g \circ F) f$ is integrable on $[a, b]$, then $g$ is integrable on $J$, and

$$
\int_{a}^{b} g(F(x)) f(x) d x=\int_{F(a)}^{F(b)} g(y) d y .
$$

Main Theorem. The above statements are all equivalent. 


$$
\text { "tmcs-tandra" — 2014/10/12 - 16:03 — page } 274-\# 6
$$

PRoOF. Our scheme of proof would be as follows:

$$
\mathrm{P} 1 \Rightarrow \mathrm{P} 2 \Rightarrow \mathrm{FTC} \Rightarrow \mathrm{CVT} 1 \Rightarrow \mathrm{CVT} 2 \Rightarrow \mathrm{P} 1
$$

$\mathrm{P} 1 \Rightarrow \mathrm{P} 2 . \quad$ Let $G(x):=\ln |F(x)|, x \in[a, b]$. Note that $G^{\prime}:=F^{\prime} / F=f / F$ a.e. Since $f$ and $1 / F$ are bounded, so is $f / F$. Let $|f / F| \leq B$, that is

$$
-B \leq \frac{f(x)}{F(x)} \leq B, \quad x \in[a, b]
$$

for some $B>0$. Let $\delta>0$ be arbitrarily fixed, and define the functions $G_{1}, G_{2}, F_{1}$, $F_{2}, f_{1}$ and $f_{2}$ on $[a, b]$ as follows:

$$
\begin{gathered}
G_{1}(x):=\frac{1}{2}(G(x)+(B+\delta) x), \quad G_{2}(x):=\frac{1}{2}(G(x)-(B+\delta) x), \\
F_{1}(x):=e^{G_{1}(x)}, \quad F_{2}(x):=e^{G_{2}(x)}
\end{gathered}
$$

and

$$
f_{1}(x):=\frac{1}{2} e^{G_{1}(x)}\left(\frac{f(x)}{F(x)}+(B+\delta)\right), \quad f_{2}(x):=\frac{1}{2} e^{G_{2}(x)}\left(\frac{f(x)}{F(x)}-(B+\delta)\right) .
$$

Note that $F$ is cotinuous and so is $G$. Hence the function $x \mapsto e^{G_{i}(x)}(i=1,2)$ is continuous, and thus integrable. Since $G^{\prime}=f / F$ a.e., it follows that $F_{1}^{\prime}=f_{1}$ a.e. and $F_{2}^{\prime}=f_{2}$ a.e., where $f_{1}$ and $f_{2}$ are both integrable on $[a, b]$, as each of them is an addition-multiplication combination of integrable functions. Equation (6) and the fact that on $[a, b]$ the exponential function is bounded from below by some positive number, yield $\left|f_{1}\right| \geq \delta_{1}$ and $\left|f_{2}\right| \geq \delta_{2}$, for some $\delta_{1}>0$ and $\delta_{2}>0$. Having $|F|=F_{1} \cdot F_{2}$, and thus differentiating both sides, gives $F^{\prime}|F| / F=F_{1}^{\prime} F_{2}+F_{1} F_{2}^{\prime}$, or $F^{\prime} / F=F_{1}^{\prime} / F_{1}+F_{2}^{\prime} / F_{2}$, and therefore $f / F=f_{1} / F_{1}+f_{2} / F_{2}$ a.e. Since $F$, hence $G$, is Lipschitz, it follows that $G_{1}$ and $G_{2}$, hence $F_{1}$ and $F_{2}$, are Lipschitz. Notice that each of the functions $f_{1} / F_{1}, f_{2} / F_{2}$, and $f / F$, is a product of two integrable functions, so it is integrable. Hence by the linearity of the integral and $\mathrm{P} 1$,

$$
\begin{aligned}
\int_{a}^{b} \frac{f(x)}{F(x)} d x & =\int_{a}^{b} \frac{f_{1}(x)}{F_{1}(x)} d x+\int_{a}^{b} \frac{f_{2}(x)}{F_{2}(x)} d x \\
& =\int_{F_{1}(a)}^{F_{1}(b)} \frac{1}{y} d y+\int_{F_{2}(a)}^{F_{2}(b)} \frac{1}{y} d y \\
& =\ln \left|F_{1}(b)\right|-\ln \left|F_{1}(a)\right|+\ln \left|F_{2}(b)\right|-\ln \left|F_{2}(a)\right| \\
& =\ln \left|F_{1}(b) \cdot F_{2}(b)\right|-\ln \left|F_{1}(a) \cdot F_{2}(a)\right|
\end{aligned}
$$




$$
\text { "tmcs-tandra" — 2014/10/12 — 16:03 — page } 275 \text { — \#7 }
$$

Equivalences of Some Forms of the Change of Variable Formula...

$$
=\ln |F(b)|-\ln |F(a)| \text {. }
$$

$\mathrm{P} 2 \Rightarrow$ FTC. Let $H(x):=e^{F(x)}, x \in[a, b]$. Let $h:=H \cdot f$, so that $f=h / H$. Since $F^{\prime}=f$ a.e., $f$ is integrable and $H$ is continuous, it follows that $H^{\prime}=h$ a.e. and $h$ is integrable. Since $F$ is Lipschitz, so is $H$. Applying P2 for the functions $h$ and $H$, we get

$$
\int_{a}^{b} f(x) d x=\int_{a}^{b} \frac{h(x)}{H(x)}=\ln |H(b)|-\ln |H(a)|=F(b)-F(a) .
$$

FTC $\Rightarrow$ CVT 1 . It suffices to consider the case where $f \geq 0$, as the other case is similar. Since $f \geq 0$, it follows from the FTC and the monotonicity of the integral that $F$ is non decreasing. Let $\epsilon>0$ be given. The integrability of $g$ on $[F(a), F(b)]$ along with the integrability of $f$ on $[a, b]$, and the uniform continuity of $F$ on $[a, b]$, while noting Theorem 1(iii)(g) and the FTC, allow us to set a partition $x_{0}:=a<x_{1}<\ldots<x_{n}:=b$ of $[a, b]$ such that for any $c_{i} \in\left[x_{i-1}, x_{i}\right]$, $i=1, \ldots, n$,

$$
\left|\sum_{i=1}^{n} g\left(F\left(c_{i}\right)\right)\left(F\left(x_{i}\right)-F\left(x_{i-1}\right)\right)-\int_{F(a)}^{F(b)} g(y) d y\right|<\epsilon
$$

and

$$
\begin{aligned}
& \sum_{i=1}^{n}\left|f\left(c_{i}\right)\left(x_{i}-x_{i-1}\right)-\int_{x_{i-1}}^{x_{i}} f(x) d x\right| \\
= & \sum_{i=1}^{n}\left|f\left(c_{i}\right)\left(x_{i}-x_{i-1}\right)-\left(F\left(x_{i}\right)-F\left(x_{i-1}\right)\right)\right|<\epsilon .
\end{aligned}
$$

Since the function $g$ is bounded, there exists $M>0$ such that $|g(y)| \leq M$, for all $y \in J$. Then (7) and (8) give

$$
\begin{aligned}
& \left|\sum_{i=1}^{n} g\left(F\left(c_{i}\right)\right) f\left(c_{i}\right)\left(x_{i}-x_{i-1}\right)-\int_{F(a)}^{F(b)} g(y) d y\right| \\
\leq & \left|\sum_{i=1}^{n} g\left(F\left(c_{i}\right)\right)\left(f\left(c_{i}\right)\left(x_{i}-x_{i-1}\right)-\left(F\left(x_{i}\right)-F\left(x_{i-1}\right)\right)\right)\right| \\
& +\left|\sum_{i=1}^{n} g\left(F\left(c_{i}\right)\right)\left(F\left(x_{i}\right)-F\left(x_{i-1}\right)\right)-\int_{F(a)}^{F(b)} g(y) d y\right|<M \epsilon+\epsilon .
\end{aligned}
$$

Therefore $(g \circ F) f$ is integrable on $[a, b]$, and (4) holds. 
CVT $1 \Rightarrow$ CVT 2. Substituting $g(y):=1$ into (4), gives $\int_{a}^{b} f(x) d x=F(b)-F(a)$, and so we have the formula

$$
\int_{s}^{t} f(x) d x=F(t)-F(s)
$$

for $s, t \in[a, b]$. Since $|f| \geq \delta$, where $f$ has the same sign on $[a, b]$, it follows that $|F(s)-F(t)|=\left|\int_{s}^{t} f(x) d x\right| \geq \delta|s-t|>0$ if $s \neq t$, showing that $F$ is one-to-one. Since $F$ is continuous on $[a, b]$, it follows that $F^{-1}$ is continuous, hence uniformly continuous, on the interval $J=F([a, b])$. It is enough to consider the case where $f \geq 0$, so that $F$, hence $F^{-1}$, is strictly increasing. Let $\epsilon>0$ be given. The integrability of $f$ and $(g \circ F) f$ on $[a, b]$, together with Theorem 1(iii)(g) and (9), allow us to choose $\eta>0$ such that if $x_{0}:=a<x_{1}<\ldots<x_{n}:=b$ is any partition of $[a, b]$ with $x_{i}-x_{i-1}<\eta$, for all $i=1, \ldots, n$, then for any $c_{i} \in\left[x_{i-1}, x_{i}\right]$,

$$
\left|\sum_{i=1}^{n} g\left(F\left(c_{i}\right)\right) f\left(c_{i}\right)\left(x_{i}-x_{i-1}\right)-\int_{a}^{b} g(F(x)) f(x) d x\right|<\epsilon
$$

and

$$
\begin{aligned}
& \sum_{i=1}^{n}\left|\int_{x_{i-1}}^{x_{i}} f(x) d x-f\left(c_{i}\right)\left(x_{i}-x_{i-1}\right)\right| \\
= & \sum_{i=1}^{n}\left|\left(F\left(x_{i}\right)-F\left(x_{i-1}\right)\right)-f\left(c_{i}\right)\left(x_{i}-x_{i-1}\right)\right|<\epsilon .
\end{aligned}
$$

Since $F^{-1}$ is uniformly continuous on $J$, there exists $\delta>0$ such that, for any $u, v \in J$, if $|u-v|<\delta$, then $\left|F^{-1}(u)-F^{-1}(u)\right|<\eta$. Let $y_{0}:=f(a)<y_{1}<$ $\ldots<y_{n}:=f(b)$ be any partition of $[F(a), F(b)]$ such that $y_{i}-y_{i-1}<\delta$, for all $i=1, \ldots, n$. Let $x_{i}:=F^{-1}\left(y_{i}\right)$, that is $y_{i}=F\left(x_{i}\right)$. Then $x_{i}-x_{i-1}<\eta$. Choose any $d_{i}:=F\left(c_{i}\right) \in\left[y_{i-1}, y_{i}\right]$. Since the function $g$ is bounded, there exists $M>0$ such that for all $y \in J,|g(y)| \leq M$. Therefore, in view of (10) and (11),

$$
\begin{aligned}
& \left|\sum_{i=1}^{n} g\left(d_{i}\right)\left(y_{i}-y_{i-1}\right)-\int_{a}^{b} g(F(x)) f(x) d x\right| \\
\leq & \left|\sum_{i=1}^{n} g\left(F\left(c_{i}\right)\right)\left(\left(F\left(x_{i}\right)-F\left(x_{i-1}\right)\right)-f\left(c_{i}\right)\left(x_{i}-x_{i-1}\right)\right)\right| \\
& +\left|\sum_{i=1}^{n} g\left(F\left(c_{i}\right)\right) f\left(c_{i}\right)\left(x_{i}-x_{i-1}\right)-\int_{a}^{b} g(F(x)) f(x) d x\right|<M \epsilon+\epsilon .
\end{aligned}
$$




$$
\text { "tmcs-tandra" — 2014/10/12 — 16:03 — page } 277 \text { — \# }
$$

Thus $g$ is integrable on $J$ and (5) is fulfilled.

CVT $2 \Rightarrow \mathrm{P} 1$. First note that a similar argument as used in early part of the proof of CVT $1 \Rightarrow$ CVT 2 implies that $F$ is strictly monotone, so that it is oneto-one. Let $D:=\left\{x \in[a, b]: F^{\prime}(x)=f(x)\right\}$. Define a function $h$ on $J:=F([a, b])$ as follows:

$$
h(F(x)):=\left\{\begin{array}{cll}
\left(F^{-1}\right)^{\prime}(F(x)) & \text { if } & x \in D \\
\frac{1}{f(x)} & \text { if } & x \in[a, b] \backslash D .
\end{array}\right.
$$

Notice that $\left(F^{-1}\right)^{\prime}(F(x))$ exists whenever $F^{\prime}(x)$ does, where each relates to the other by the formula $\left(F^{-1}\right)^{\prime}(F(x)) F^{\prime}(x)=1$. Thus

$$
h(F(x)) f(x)=\left(F^{-1}\right)^{\prime}(F(x)) f(x)=1,
$$

for all $x \in D$. It then follows from the definition of $h$ that $h(F(x)) f(x)=1$, for all $x \in[a, b]$. Since $|f| \geq \delta>0$, it follows that $h$ is bounded. As a constant function, $(h \circ F) f$ is integrable, and so by CVT $2, h$ is integrable. Since $f$ has the same sign on $[a, b]$, it follows that $|F(s)-F(t)|=\left|\int_{s}^{t} f(x) d x\right| \geq \delta|s-t|$, for all $s, t \in[a, b]$, showing that $F^{-1}$ is Lipschitz. Since $F^{\prime}=f$ a.e on $[a, b]$, it follows that $[a, b] \backslash D$ is of measure 0 . The fact that $F$ is Lipschitz, by Theorem 1(i)(f), implies that $F([a, b] \backslash D)=\{F(x): x \in[a, b] \backslash D\}$ is of measure 0 . It then follows from the definition of $h$ that $\left(F^{-1}\right)^{\prime}=h$ a.e. on $J$. Since $f$ is integrable, it is bounded. Consequently, since $(h \circ F) f=1$, we then have $|h| \geq \delta_{0}$, for some $\delta_{0}>0$. Now let $g(x):=f(x) / F(x), x \in[a, b]$. Then $g$ is integrable, and $g\left(F^{-1}(y)\right) h(y)=1 / y$, for all $y \in J$. As a continuous function, $\left(g \circ F^{-1}\right) h$ is integrable on $J$, where $J=[F(a), F(b)]$ if $F$ is increasing, and $J=[F(b), F(a)]$ if $F$ is decreasing, and hence by CVT 2,

$$
\int_{F(a)}^{F(b)} g\left(F^{-1}(y)\right) h(y) d y=\int_{F^{-1}(F(a))}^{F^{-1}(F(b))} g(x) d x
$$

that is $\int_{F(a)}^{F(b)} 1 / y d y=\int_{a}^{b} f(x) / F(x) d x$. This proves part (i). Part (ii) is immediate by substituting $f(x):=\ln |x|$ and $g(y):=1$ into (5).

REMARK. We conclude our discussion with some interesting remarks from Main Theorem:

- For CVT 1 (or equivalently CVT 2 or FTC) to be true it is enough to assume two particular formulas obtained from CVT 1:

$$
\int_{a}^{b} \frac{f(x)}{F(x)} d x=\int_{F(a)}^{F(b)} \frac{1}{y} d y
$$


where $F$ is Lipschitz, non zero, $F^{\prime}=f$ a.e., with $f$ is integrable on $[a, b]$, $|f| \geq \delta$ for some $\delta>0$, and $f$ has the same sign on $[a, b]$ (which is obtained from $C V T 1$ by setting $g(y):=1 / y)$, and

$$
\int_{a}^{b} \frac{1}{x} d x=\int_{\ln |a|}^{\ln |b|} d y
$$

(which is obtained from CVT 1 by setting $f(x):=\ln |x|$ and $g(y):=1$ ).

- For FTC (or equivalently CVT 1 or CVT 2) to be true it is enough to assume two particular formulas obtained from the FTC:

$$
\int_{a}^{b} \frac{1}{x} d x=\ln |b|-\ln |a|
$$

and

$$
\int_{a}^{b} \frac{h(x)}{H(x)} d x=\int_{H(a)}^{H(b)} \frac{1}{y} d y
$$

where $H$ is Lipschitz, non zero, $H^{\prime}=h$ a.e., with $h$ is integrable on $[a, b]$, $|h| \geq \delta$ for some $\delta>0$, and $h$ has the same sign on $[a, b]$. Notice that equation (15) can be obtained from the FTC by setting $f(x):=\int_{H(a)}^{H(x)} 1 / y d y$, where

$$
f^{\prime}(x)=\frac{d}{d x}\left(\int_{H(a)}^{H(x)} \frac{1}{y} d y\right)=\frac{H^{\prime}(x)}{H(x)}
$$

by the chain rule for derivatives, while noting (14) and $d(\ln |t|) / d t=1 / t$. Here $H^{\prime} / H=h / H$ a.e., where $h / H$ is integrable on $[a, b]$, as it is the product of two integrable functions $h$ and $1 / H$ on $[a, b]$.

- For CVT 1, CVT 2, and the FTC to be true it is enough to assume the formula

$$
\int_{a}^{b} \frac{f^{\prime}(x)}{f(x)} d x=\ln |f(b)|-\ln |f(a)|=\int_{\ln |f(a)|}^{\ln |f(b)|} d y
$$

Acknowledgment

The author is very grateful to the referees for their valuable advices.

\section{References}

[1] R. G. Bartle, A Modern Theory of Integration, Oxford University Press, Providence, RI, 2001. 


$$
\text { "tmcs-tandra" — 2014/10/12 — 16:03 — page 279 — \#11 }
$$

[2] M. W. Botsko, A fundamental theorem of calculus that applies for all Riemann integrable functions, Math. Mag. 64 (1991), 347-348.

[3] E. Hewitt and K. Stromberg, Real and Abstract Analysis, A Modern Treatment of the Theory of Functions of a Real Variable, Springer-Verlag, New York, 1975.

[4] H. Kestelman, Change of variable in Riemann integration, Math. Gaz. 45 (1961), $17-23$.

[5] D. Preiss and J. Uher, A remark on the subtitution for the Riemann integral, Casopis Pěst. Mat. 95 (1970), 345-347.

[6] H. L. Royden, Real Analysis, Prentice-Hall, Inc., 1988.

[7] W. Rudin, Principles of Mathematical Analysis, McGraw-Hill, Inc., New York, 1976.

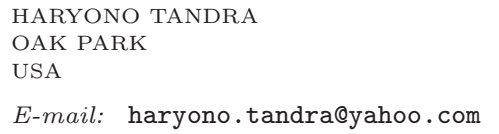

(Received May, 2014) 